\title{
Femoral shaft fractures in children treated with elastic nail in general hospital in Kingdom of Saudi Arabia
}

\author{
Mohammad Khair Alawir, Stefan Cristea \\ ${ }^{1}$ Department of Orthopaedics and Traumatology, Bader General Hospital, Almadina, \\ Kingdom of Saudi Arabia \\ 2Department of Orthopaedics and Traumatology, Emergency Hospital of Saint Pantelimon, \\ Bucharest, Romania

\begin{abstract}
Objective. To evaluate the outcome of TEN for the treatment of fracture of shaft of femur in pediatric age group.

Method. 140 patients with diaphyseal femoral fracture were treated with retrograde Titanium elastic nail the age group 6-14 years. Two nails with same diameter were used in each fracture. The final result were evaluated using the criteria of Flynn et al.

Results. The results were $102(72.8 \%)$ excellent, $34(24.2 \%)$ good, and $6(3 \%)$ poor. Using the criteria of Flynn et al. All fractures were radiographically united at a median of $9(6-11) \mathrm{w}$. The nails were removed after a median of $12 \mathrm{M}$ postoperatively. The most common complication was irritation at nail insertion.

Conclusion. TEN is an reliable, safe, and cost-effective implant for pediatric femoral fracture and it is an economic synthesis. It is relatively free of complication.
\end{abstract}

Keywords: children, nail, femoral shaft, fracture, treatment

\author{
Abbreviations: \\ TEN - Titanium Elastic Nail \\ W-week \\ M - month \\ KSA - Kingdom of Saudi Arabia \\ GA - General Anesthesia
}

\section{INTRODUCTION}

Fractures of the femoral shaft are common in children. Over the past few decades, children femoral fractures have been more appropriately managed with operative treatment rather than conservative treatment because the former has more rapid recovery and shorter immobilization (1). In pediatric femoral shaft fractures the aim of treatment is to stabilize the fracture, maintain the length, control the rotational alignment, promote healing, and minimize the morbidity and complications for the child. The treatment options are age related, depends on the type of injury, associated injuries and the location and type of fracture. Small children less than five years old are treated with early reduction and hip spica while young adolescents more than 15 years old are treated with intramedullary interlocking nail. for children between 6 and 14 years there are surgical and nonsurgical options, early spica casting, and casting, plating, external fixation, and flexible intermedullary nails. TEN becomes the treatment of choice for femoral fracture in that group of age due to excellent results and lack of serious complications $(2,3)$.

Each modality of fixation has its own set of complications. External fixations for example, have been associated with pin-tract infection and refracture (4), whereas traction leads to prolonged immobilization (5). Plate osteosynthesis has the potential to expose the child to an extensive scar related to larger incision and extensive soft tissue stripping (6), and locked nailing may potentially 
damage the physis (7). Elastic stable intramedullary nails has its own set of problems including nail irritation, refracture, and superficial and deep infections. The most common complication was irritation at nail insertion.TEN intervention results in a shorter hospital stay and has economic and social benefits over conservative treatment. But this procedure requires a $\mathrm{C}$-arm facility, which is not present at all centers and causes radiation exposure $(8,9)$. TEN don't disturb the fracture hematoma when done as closed procedure, so chance of infection is very less.

This study aims to evaluate the outcome of TEN for the treatment of fracture of shaft of femur in pediatric age group.

\section{MATERIAL AND METHODS}

This study was conducted in BADER General Hospital in Kingdom of Saudi Arabia, 140 patient of femoral shaft fractures treated with titanium elastic nail between 2011 and 2017 with follow up period of 2 years. We excluded open fractures, underlying neuromuscular disorder, pathological fractures, metabolic bone disorder. Indication for surgery was displaced femoral shaft fracture in the age group 6-14 years. Consent was obtained from patient family for inclusion in this study. There were 98 males and 42 females. Fracture location were 92 midshaft, 30 proximal, and 18 distal fractures. Fracture patterns included 96 transverse, and 44 oblique. Associated injuries were present in 42patients 22 had head injuries, and 18 had fracture of other bones like forearm. Preoperatively patients were placed in Thomas Knee splint with below knee skin traction. Two titanium nails (Fig. 1, 2) were inserted across the fracture in a retrograde technique, but sometimes we use 3 nails, the nail was driven towards the femoral neck approximately $2 \mathrm{~cm}$ distal to the capital physis and the other towards the greater trochanter, $1 \mathrm{~cm}$ distal to its physis under fluoroscopy. 10, each Nail diameter must be able to fill $40 \%$ of the narrowest canal. We measured the internal diameter of the femoral diaphysealby antero-posterior and lateral roentgenogram but intraoperative decision was taken by operation surgeon.

Both plaster and stitches were removed 2 weeks after surgery. Partial weight bearing was started 2 weeks after surgery in cases of midshaft transverse fractures and delayed to 4 weeks in cases of oblique and comminuted fractures. All patients were followed radiographically until the fracture healed, and were reviewed clinically for leg-length discrepancy, rotational and angular deformities.

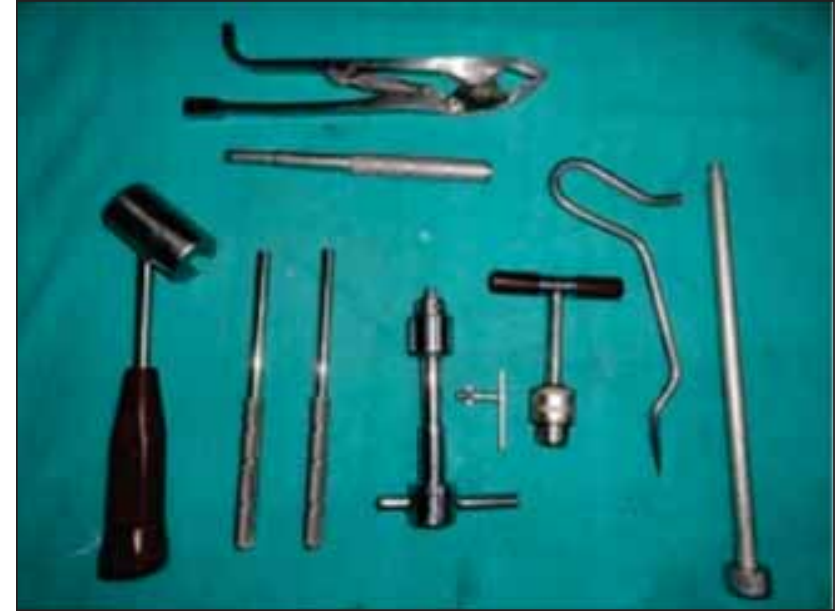

FIGURE 1. TEN instruments, it is consist of: Awl, Inserter, Extraction pliers, Cutter, and Impactor

FIGURE 2. TEN, it is available in six diameter, $1.5 \mathrm{~mm}$ to $4 \mathrm{~mm}$

\section{SURGICAL TECHNIQUE}

Under general anaesthesia patients were placed on an orthopaedic fracture table and a reduction of the fracture by traction guided by fluoroscopy was done. Two and sometimes 3 till the medullary canal was fulfill, retrograde titanium elastic nails were used in all pediatric femoral shaft fractures. A traction table with a "C-arm" image intensifier was used for older children. Closed reduction was performed by manual traction and gentle rotation along with use of F-tool.

By using lateral and medial incisions above the knee, the nails penetrate the medullary space and avoid the distal femoral epiphyseal line, starting 2 $\mathrm{cm}$ proximal to the distal femoral epiphyseal plate. A nail loaded onto a $\mathrm{T}$ handle was then inserted through the entry point into the medullary canal by rotator movements of the wrist and advanced upto fracture site, initially at a right angle and then at 45 degrees, inclined away from the distal femoral epiphysis. Check AP and lateral views to ensure proper placement of the nail.

The nail diameter chosen was $40 \%$ of the narrowest canal diameter, determined preoperatively. The two nails must have similar diameter (Fig. 3). Nails come in five diameters from $2.5 \mathrm{~mm}$ to 4.5 $\mathrm{mm}$ in fixed length. 

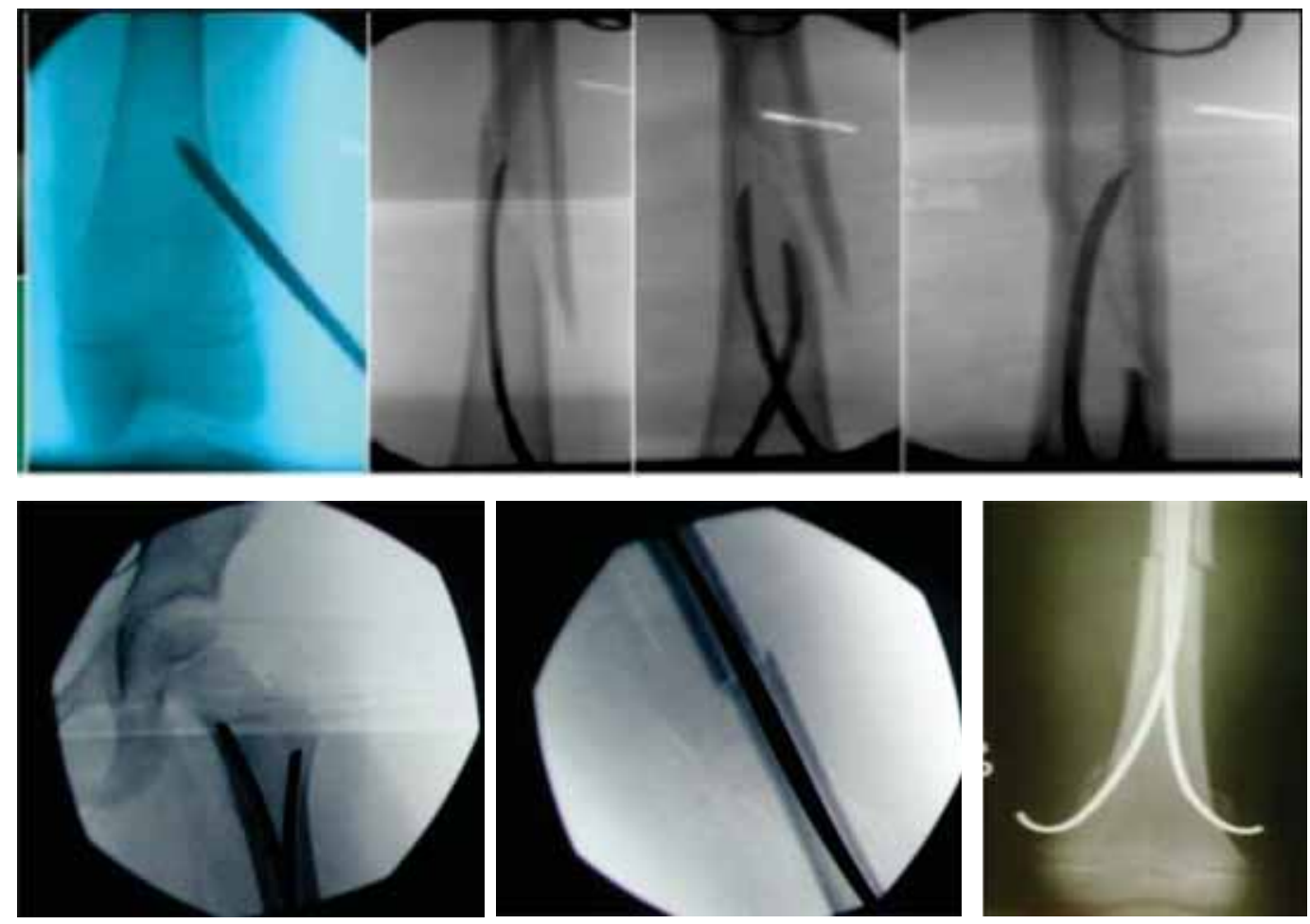

FIGURE 3. Operative technique, starting $2 \mathrm{~cm}$ proximal to the distal femoral epiphyseal plate. Two titanium nails $(2.5 \mathrm{~mm})$ were inserted across the fracture in a retrograde technique.

Confirm fracture alignment, rotation, and nail position by XRY. The second nail was introduced from the opposite cortex up to the proximal fragment, Traction was released during crossing of second nail at the fracture site, to avoid any distraction. Care was taken to see that the nails did not cross the epiphyseal growth plate proximally and that both nails were at the same level proximally. Attention was given to avoid short nails, Nails were left protruding about $0.5-1.0 \mathrm{~cm}$ at the distal end for easy removal later on (10).

Postoperatively, sutures were removed on the 12th postoperative day. Only unstable fractures were immobilized for 4-6 weeks to provide extra stability fractures, the patients were kept in bed with knee flexion and extension permitted. Full weight-bearing was permitted only on fracture union, between 6-9 weeks. Patients were followed up at regular intervals of 7 days after surgery, 6 weeks, 3 months, 1 year, 2 year. At each visit patient were clinically and radiological evaluated. At follow-up, all patients had unrestricted movement of the hip and knee joints.

\section{RESULTS}

All 140 fractures healed (Fig. 4). All children had closed fractures. Children who were excluded from the study were those with open fractures or those with pathological fractures, the union aver- age was 8.7 weeks. The right femur was affected in 75 children $(56 \%)$, whereas the left femur was affected in 65 children (44\%). All were acute traumatic fractures. 104 fractures were caused by minor falls (e.g. during playing, sliding, or cycling). 36 children were struck by motor vehicles as pedestrians. The median duration of surgery was 67.5 (45-90) minutes, No case of non-union, 3 case delayed union. The mean hospital stay was 3.1 days. Closed reduction was achieved in 122 cases whereas open reduction was achieved in 18 cases. Full weight-bearing was start at $6-10$ weeks. The time to return to preoperative level of activity average was 4.2 months, return to school was with an average of 9.6 weeks.

Removal was done under GA after achieving solid union at the end of one year. 1 patient had the nails removed at 8 weeks because they penetrated the skin, and no refractures was observed after nail removal. All fractures were evaluated using the criteria of Flynn et al.

\section{COMPLICATIONS}

The most common complication was irritation at nail insertion leading to bursitis in 12 patient (Fig. 5), and this complication resolved after nail removal. To reduce this complication leave approximately 1 to $1.5 \mathrm{~cm}$ of the nail outside the medullary canal (11), with the nail ends flush with and 


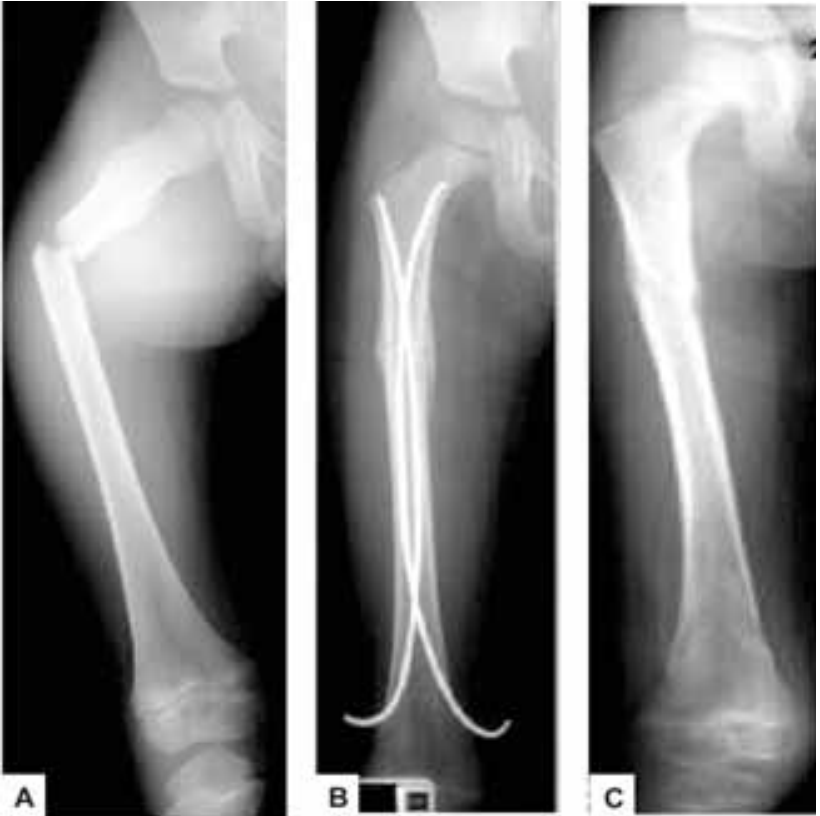

FIGURE 4. The outcome of elastic nail for the treatment of fracture of shaft of femur in pediatric aged 11 years: A-preoperative, $B$-postoperative, $C$ - after nail removal.

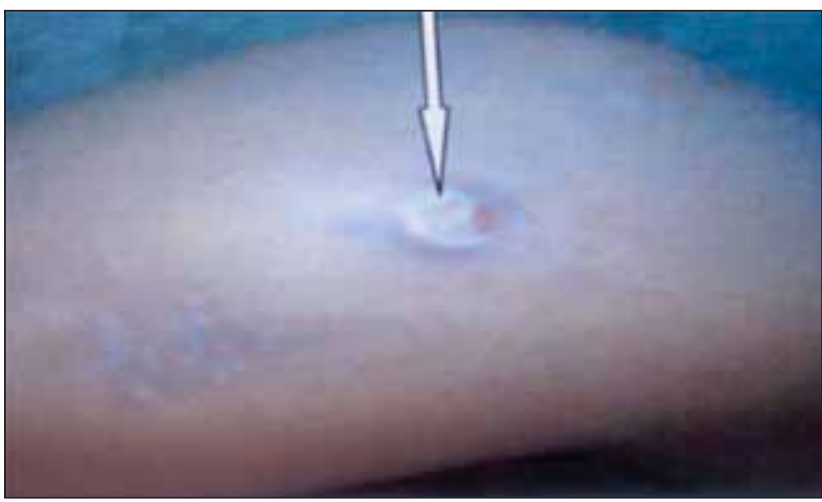

FIGURE 5. Irritation at nail insertion, skin irritation dead to bursitis (arrow), treated by nail removal

parallel to the metaphysis. The nail should not be bent at the end (12). Modified nail cutters prevent oblique nail cuts and sharp edges. Possible treatments for prominent nails include early removal, trimming of the nail end, advancement of the nail, exchange nailing, or observation.

Limb length discrepancy [LLD] were seen in $32 \%$ but LLD greater than $1 \mathrm{~cm}$ occurs in $15 \%$ but it decreased to $5 \%$ by 2 years. No case of non-union, and 6 case delayed union. Minor malalignment was observed in 24 cases, whereas significant malalignment was observed in only 4 cases. Rotational malalignment was observed in 7 cases. The main cause of angular malalignment was comminuted fractures.

Only 2 case of superficial infection was observed and 1case of deep infection treated with antibiotics, and no case of osteomyelitis. Migration of the nail was not seen in this study, 12 cases had limitation in last 22 degree of knee flexion due to nail ends, and it was improved after nail removal and physiotherapy. The nail were removed with an average of 12 months with no complication. No case of perforation of cortex of femoral neck.

The results were 102 excellent, 34 good, and 6 poor, using the criteria of Flynn et al. The surgeon recommended post-operative immobilisation in a cast in 30 fractures and a knee immobiliser in 86 fractures $(62.4 \%)$. No case of compartment syndrome, haematoma, and blood loss. No complications were associated with the nail removal procedure and no refractures was observed after nail removal.

TABLE 1. Complications of TEN

\begin{tabular}{|l|c|}
\hline Complications & $\begin{array}{c}\text { fractures No } \\
\text { total } \mathbf{1 4 0}(\%)\end{array}$ \\
\hline Nonunion & 0 \\
\hline Migration of the nail & 0 \\
\hline Malalignment & $4(2.8)$ \\
\hline Deep infection & $1(0.7)$ \\
\hline Blood loss & 0 \\
\hline loss of reduction & $3(2.1)$ \\
\hline Leg-length discrepancy & $7(5)$ \\
\hline Deep infection & $1(0.7)$ \\
\hline Haematoma & 0 \\
\hline Refractures after nail removal & 0 \\
\hline Irritation at the nail entry & $22(16.2)$ \\
\hline Compartment syndrome & 0 \\
\hline Penetration of proximal cortex & 0 \\
\hline Osteomyelitis & 0 \\
\hline Inflammatory reaction due to nails & $5(3.5)$ \\
\hline Superficial infection at site of nail insertion & $2(1.4)$ \\
\hline Delayed union & $3(2.1)$ \\
\hline Restricted motion knee & $12(8.5)$ \\
\hline Improper size selection & $2(1.4)$ \\
\hline Total & $62(44.2)$ \\
\hline
\end{tabular}

\section{DISCUSSION}

The ideal treatment for pediatric femoral shaft fractures depends upon the age of child, location and type of fracture, availability of facilities, knowledge of surgeon, and financial conditions to some extent (13).

Surgical treatment of femoral shaft fractures is seldom considered in patients less than 4 years of age, in whom early reduction and immobilization in a spica cast or traction are well-established methods. However, in older children, such treatment may be necessary to avert complications,such as delayed union, malunion, rotational deformity, refracture, knee stiffness, limb-length discrepancy and psychosocial problems. 
Management of femoral diaphyseal fractures in the age group of 6-15 years is controversial. There has been a resurgence worldwide for operative fixation in general and TENS in particular. Operative treatment includes plate fixation, external fixation, and flexible nailing Enders nail and TEN. Operative intervention results in a shorter hospital stay and has economic and social benefits over conservative treatment. Titanium elastic nail is a simple, load sharing, and allows mobilization and maintenance of alignment (14). Ligier et al. (1988) reported good results with elastic stable intramedullary titanium nailing of 123 femoral shaft fractures in children. They reported no nonunions or delayed unions, only 1 infection and minimal limb-length discrepancy.

TEN indicates for children of group 6-14 years with femoral shaft fractures and open physis. The indication of TEN is expanding, as their advantages are realized and complications are very less compared to other methods of fixation. The main advantages are: they are readily available in different diameters and inexpensive.

During insertion of nails, reaming is not done, and nutrient vessels are preserved, so there is a theoretical advantage of early healing of fractures (15). Children who had transverse fracture pattern had a shorter union time and was found to be heavier when compared to others. Presence of parents in the hospital reduces their working hours and increases the economic burden of the family.

From a cost-analysis standpoint, some studies suggested that the overall cost was significantly lower when TEN was used compared with traction, followed by spica casting (16).

Another study showed that patients treated with TEN were able to ambulate sooner after surgery and returned to school earlier than patients treated with traction, followed by spica casting (15). We have had a similar experience in our own practice in terms of early ambulation and early return to school. Diameter of each nail should measure $40 \%$ of the narrowest diameter of the medullary canal (16). The two nails should be of same thickness (19).

TEN when applied in retrograde manner there is little chance of avascular necrosis of femoral head
$(20,21)$. We agreed that intramedullary nail could not effectively control the torsion and shift of the fragments, particularly in cases of spiral and comminuted fractures. In these types of cases, we prolong the duration of posterior slab until fracture becomes stable. The degree of shifting of fragments caused by nail during surgery does influence the outcome of fracture healing a lot.

Comminuted fracture is the main cause for limb length discrepancy but it is insignificant complication clinically because LLD was within $1 \mathrm{~cm}$ only .Shortening of more than $1 \mathrm{~cm}$ was observed in 6 patients only, and all have comminuted fracture.

This method provides a combination of axial stability and elastic mobility that stimulates the development of bone callus. Partial rotational stability is achieved by the double crossing of the curved nails, which provides three-point fixation (Ligier et al., 1988). Stability enables rapid return to function and partial weight-bearing.

Preoperative planning, intraoperative details, and technical execution as well as appropriate postoperative care and follow-up are all important in predicting good outcomes. On account of the risk of infection as documented by the systematic review of the literature, we would recommend the use of routine perioperative antibiotics. While cutting the ends of the nails, one should be careful not to make the nails too prominent to decrease the rate of early removal and nail migration. Parents should be advised that minor complications are not uncommon, and that implant removal may be necessary particularly when symptomatic.

\section{CONCLUSION}

Titanium elastic nailing (TEN) is currently the most popular method for treating closed femoral shaft fractures in pediatric patients (6-14 years old). It is a simple, minimally invasive operation. This technique can provide a rapid recovery, short rehabilitation and decreased the hospital stay. It achieves excellent or good results in majority of patients with low complication rate, and it is an economic synthesis.

Conflict of interest: none declared Financial support: none declared

\section{REFERENCES}

1. T.P. Carey, R.D. Galpin. Flexible intramedullary nail fixation of pediatric femoral fractures Clin Orthop Relat Res, 332 (1996), pp. 110-118.

2. Flynn J.M., Hresko T., Reynolds R.A., Blasier R.D., Davidson R., Kasser J. Titanium elastic nails for pediatric femur fractures: a multicenter study of early results with analysis of complications. $J$ PediatrOrthop. 2001, 21 (1): 4-8. 10.1097/01241398-200101000-0000 
3. Heinrich S.D., Drvaric D.M., Darr K., MacEwen G.D. The operative stabilization of pediatric diaphyseal femur fractures with flexible intramedullary nails: a prospective analysis. J Pediatr Orthop, 2004, 14(4):501-

4. Aronson J., Tursky E.A. External fixation of femur fractures in children. J Pediatr Orthop 1992; 12:157-16

5. Nork S.E., Hoffinger S.A. Skeletal traction versus external fixation for pediatric femoral shaft fractures: a comparison of hospital costs and charges. J Orthop Trauma 1998; 12:563-568.

6. Kanlic E.M., Anglen J.O., Smith D.G., Morgan S.J., Pesantez R.F. Advantages of submuscular bridge plating for complex pediatric femur fractures. Clin Orthop Relat Res 2004; 426:244-251.

7. Gordon J.E., Swenning T.A., Burd T.A., Szymanski D.A., Schoenecker P.L. Proximal femoral radiographic changes after lateral transtrochanteric intramedullary nail placement in children. J Bone Joint Surg Am 2003; 85-A:1295-1301.

8. Bali K., Sudesh P., Patel S., Kumar V., Saini U., Dhillon M.S. Pediatric femoral neck fractures: Our 10 Years of Experience. Clin Orthop Surg 2011;3:302-8.

9. Pulate A., Jadhav A., Shah N.B. Study of the outcome of titanium elastic nail system in diaphyseal femoral fractures in children. J Maharashtra Orthop Assoc 2012;7:6-8.

10. Kasser J.R., Beaty J.H. Femoral shaft fracture, In R.W. Bucholz, J.D. Heckman, C.M. Court-Brown (Eds.), Rockwood \& Green's Fractures in Children (6th ed.), Lippincott Williams \& Wilkins, Philadelphia (2006), pp. 912-929

11. Sankar W.N., Jones K.J., David Horn B., Wells L. Titanium elastic nails for pediatric tibial shaft fractures. J Child Orthop. 2007 Nov;1(5):281-6. Epub 2007 Oct 17.

12. Narayanan U.G., Hyman J.E., Wainwright A.M., Rang M., Alman B.A. Complications of elastic stable intramedullary nail fixation of pediatric femoral fractures, and how to avoid them. J Pediatr Orthop. 2004 Jul-Aug;24(4):363-9.

13. L. Staheli, G. Sheridan. Early spica cast management of femoral shaft fractures in young children. A technique utilizing bilateral fixed skin traction Clin Orthop Relat Res, 126 (1977), pp. 162-166

14. Baldwin K., Hsu J.E., Wenger D.R., Hosalkar H.S. Treatment of femur fractures in school-aged children using elastic stable intramedullary nailing: A systematic review. J Pediatr Orthop B 2011; 20:303-308.

15. Pankovich A.M., Goldflies M.L., Pearson R.L. Closed Ender nailing of femoral-shaft fractures, J Bone Joint Surg Am, 61 (1979), pp. 222-232, Cross Ref View Record in Scopus

16. Buechsenschuetz K.E., MehIman C.T., Shaw K.J., Crawford A.H., Immerman E.B. Femoral shaft fractures in children: Traction and casting versus elastic stable intramedullary nailing. J Trauma 2002; 53:914-921.

17. Flynn J.M., Luedtke L.M., Ganley T.J., Dawson J., Davidson R.S., Dormans J.P. et al. Comparison of titanium elastic nails with traction and a spica cast to treat femoral fractures in children. J Bone Joint Surg Am 2004; 86-A:770-777.

18. Slongo T.F. Complication and failures of the ESIN technique. Injury. 2015; 36: S-A78-S-A85.

19. Schmittenbecher P.P., Dietz H.G., Linhart W.E. et al. Complications and problems in intramedullary nailing of children's fractures. Eur $\mathrm{J}$ Trauma. 2010; 26: 287-93

20. Bhaskar A. Treatment of long bone fractures in children by flexible titanium nails. Indian J Orthop. 2005; 39(3):166-8.

21. Lascombes P, Haumont T, Journeau P. Use and abuse of flexible intramedullary nailing in children and adolescents. J Pediatr Orthop. 2006; 26(6):827-34. 